\title{
Spironolactone use and renal toxicity: population based longitudinal analysis
}

\author{
Li Wei, lecturer, ${ }^{1}$ Allan D Struthers, professor, ${ }^{2}$ Tom Fahey, professor, ${ }^{3}$ Alexander D Watson, general \\ practitioner, ${ }^{4}$ Thomas M MacDonald, professor $^{1}$
}

${ }^{1}$ Medicines Monitoring Unit (MEMO), Division of Medical Sciences, Ninewells Hospital and Medical School, Dundee DD1 9SY

${ }^{2}$ Clinical Pharmacology and Therapeutics, Division of Medical Sciences, Ninewells Hospital and Medical School

${ }^{3}$ Department of General Practice, Royal College of Surgeons in Ireland, Mercer's Medical Centre, Dublin 2, Ireland

${ }^{4}$ Westgate Health Centre, Dundee DD2 4AD

Correspondence to: T MacDonald t.m.macdonald@dundee.ac.uk

Cite this as: $B M J$ 2010;340:c1768 doi:10.1136/bmj.c1768

\author{
ABSTRACT \\ Objective To determine the safety of spironolactone \\ prescribing in the setting of the UK National Health \\ Service. \\ Design Population based longitudinal analysis using a \\ record linkage database. \\ Setting Tayside, Scotland. \\ Population All patients who received one or more \\ dispensed prescriptions for spironolactone between \\ 1994 and 2007.
}

Main outcome measures Rates of prescribing for spironolactone, hospital admissions for hyperkalaemia, and hyperkalaemia and renal function without admission, before and after the publication of results from the Randomised Aldactone Evaluation Study (RALES). Results Prescriptions for spironolactone and measurements of serum creatinine and serum potassium all increased in parallel in Tayside after the release of the RALES results in 1999 (from 2847, 5345, and 5246 in the first half of 1999 to 6582,10753 , and 10534 by the second half of 2001 , and to 8619,17844 , and 17649 by 2007). These increases occurred in patients with and without heart failure. Few hospital admissions for hyperkalaemia occurred over this time: three in the first quarter of 1995, two in the last quarter of 2001, and three in 2007. Among patients who were taking angiotensin converting enzyme inhibitors and who had recently been admitted to hospital for heart failure, the rate of spironolactone use was 19.8 per 100 patients in early 1999 rising to 70.1 per 100 patients by late $2001(\mathrm{P}<0.01)$ and 61.3 by 2007 . The rate of outpatient measured hyperkalaemia (serum $\mathrm{K}^{+}>6 \mathrm{mmol} / \mathrm{l}$ ) did not increase over time (9.9 per 100 patients in early 1999, 6.9 per 100 patients in late 2001, and 2.9 per 100 patients in 2007) despite the increased use of spironolactone.

Conclusions Despite a marked increased in the use of spironolactone in patients with and without heart failure, no increase was seen in hospital admissions for hyperkalaemia and outpatient hyperkalaemia actually fell. Careful monitoring of patients prescribed spironolactone seems to have been associated with no increase in risk of hyperkalaemia.

\section{INTRODUCTION}

The mineralocorticoid antagonist spironolactone is efficacious in patients with heart failure. ${ }^{1}$ It is also effective in patients with resistant hypertension, primary aldosteronism, and cirrhosis with secondary aldosteronism..$^{2-4} \mathrm{~A}$ recent study showed that a rapid increase in use of spironolactone occurred in Canadian patients with heart failure concurrently treated with an angiotensin converting enzyme (ACE) inhibitor after the publication of the Randomised Aldactone Evaluation Study (RALES).${ }^{15}$ However, a marked concurrent increase in hospital admissions with (and subsequent deaths from) hyperkalaemia also occurred. We believed that the risk associated with use of spironolactone was managed better in our region (Tayside) than in Canada and that serious adverse effects were very rare. To test whether we had similar adverse effects to those seen in Canada, we did a population based study linking the dispensed prescribing of spironolactone with hospital admissions for hyperkalaemia and renal failure and results of biochemical monitoring in outpatients.

\section{METHODS}

We did this study in the population of Tayside in Scotland, using the MEMO (Medicines Monitoring Unit) record linkage database. ${ }^{6}$ The MEMO database covers a geographically compact population and serves about 400000 patients in the National Health Service in Scotland, $97 \%$ of whom are white. In brief, this database contains several datasets including all dispensed community prescriptions, hospital discharge data, and other data that are linked by a unique patient identifier, the community health index number. The data have been validated and made anonymous for the purposes of research, as approved by the government appointed guardians of patient confidentiality.

\section{Study design and population}

This was a population based longitudinal analysis using the MEMO record linkage database in people resident in Tayside and registered with a general practitioner between January 1994 and December 2007. We studied patients who were treated with spironolactone between January 1994 and December 2001. We compared the 

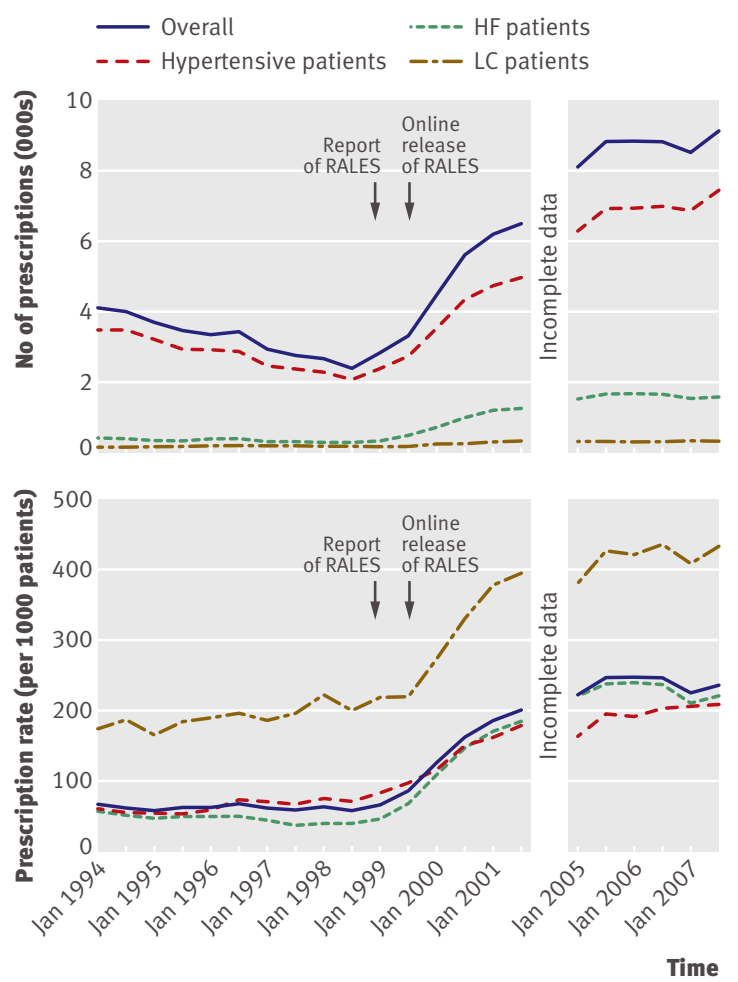

Fig 1| Spironolactone prescriptions (top) and prescribing rates (bottom) for different indications in Tayside population between 1994 and 2007. HF=heart failure; LC=liver cirrhosis

data on spironolactone prescriptions and serum creatinine/potassium measurements before and after the date of online release of the RALES results. This period of time matched that of the Canadian study, ${ }^{3}$ which enabled us to compare the two studies. We did a further analysis between 2005 and 2007 to determine whether these trends persisted. For technical reasons, the data were incomplete between 2002 and 2004 .

\section{Definitions}

We defined patients with heart failure as those who had a hospital admission for heart failure with an ICD-9 (international classification of diseases, 9th revision) diagnostic code of 428 or an ICD-10 code of I50. We defined patients with liver cirrhosis as those who had a hospital admission for liver failure with an ICD-9 code of 571 or an ICD-10 code of K70 or K74. Patients with hypertension were those who were on spironolactone treatment and who did not have a diagnosis of heart failure or liver cirrhosis (presumed to be mostly hypertensive patients).

Biochemical data were available for the entire region (primary and secondary care) from 1995 onwards. We defined spironolactone associated hyperkalaemia as serum potassium greater than $6 \mathrm{mmol} / \mathrm{l}$ in a patient taking spironolactone treatment.

\section{Outcome variables}

The outcome measures were rates of prescribing for spironolactone, any hospital admission for hyperkalaemia, any outpatient serum potassium above $6 \mathrm{mmol} / \mathrm{l}$, and serum creatinine above $220 \mu \mathrm{mol} / \mathrm{l}$.

\section{Statistical analysis}

We used a six month interval in the time series data for spironolactone prescriptions and serum measurements of creatinine and potassium. We used a regression model that takes into account autocorrelation between the measures over time to assess whether associations existed between spironolactone prescribing and biochemistry measurements. We examined rates of spironolactone prescriptions, hospital admissions for hyperkalaemia, and serum measurements for creatinine and potassium before and after the release of the RALES study results. We did a trend test to check whether spironolactone prescriptions and measurements of serum creatinine/potassium increased over the entire study period. We compared hyperkalaemia associated with spironolactone prescribing between patients with heart failure, liver cirrhosis, and hypertension. We summarised data as mean (SD) or median (interquartile range) for continuous variables and number (percentage) of patients for categorical variables. We did $\chi^{2}$ tests and Kruskal-Wallis tests to determine significant differences in patients' characteristics between the three groups. We used SAS software (version 9.1) for all statistical analyses.

\section{RESULTS}

Spironolactone use in Tayside population

The number of spironolactone prescriptions and the number of patients who were taking spironolactone rose significantly after the release of the RALES study results (September 1999) from 1018 patients with 2847 prescriptions in the first half of 1999 to 2067 patients with 6582 prescriptions by the second half of 2001. We also saw an increase in recent years, with 2564 patients and 8619 prescriptions in the first half of 2007 (fig 1). Tayside has a very stable population, so prescription numbers reflect the rate of prescribing. The proportions of patients who were taking low dose ( $\leq 25 \mathrm{mg}$ /day), medium dose (26-50 mg/day), and high dose $(>50 \mathrm{mg} /$ day) spironolactone were $50.8 \%, 24.9 \%$, and $24.3 \%$ for patients with heart failure $(\mathrm{n}=1235) ; 6.5 \%, 15.9 \%$, and $77.6 \%$ for patients with liver cirrhosis $(n=340)$; and 33.9\%, 32.8\%, and 33.3\% for patients with hypertension $(\mathrm{n}=5883)$ during the period 1994-2001.

\section{Serum measurements and hospital admissions}

Measurements of serum creatinine and serum potassium increased in parallel in patients who were taking spironolactone in Tayside after the release of the RALES results in 1999 (from 5345 and 5246 in the first half of 1999 to 10753 and 10534 by the second half of 2001). These increases before and after July 1999 were statistically significant $(\mathrm{P}<0.01)$. These increases occurred in patients with and without heart failure and continued in recent years (to 17844 and 17649 in 2007). The regression coefficient was 2.18 

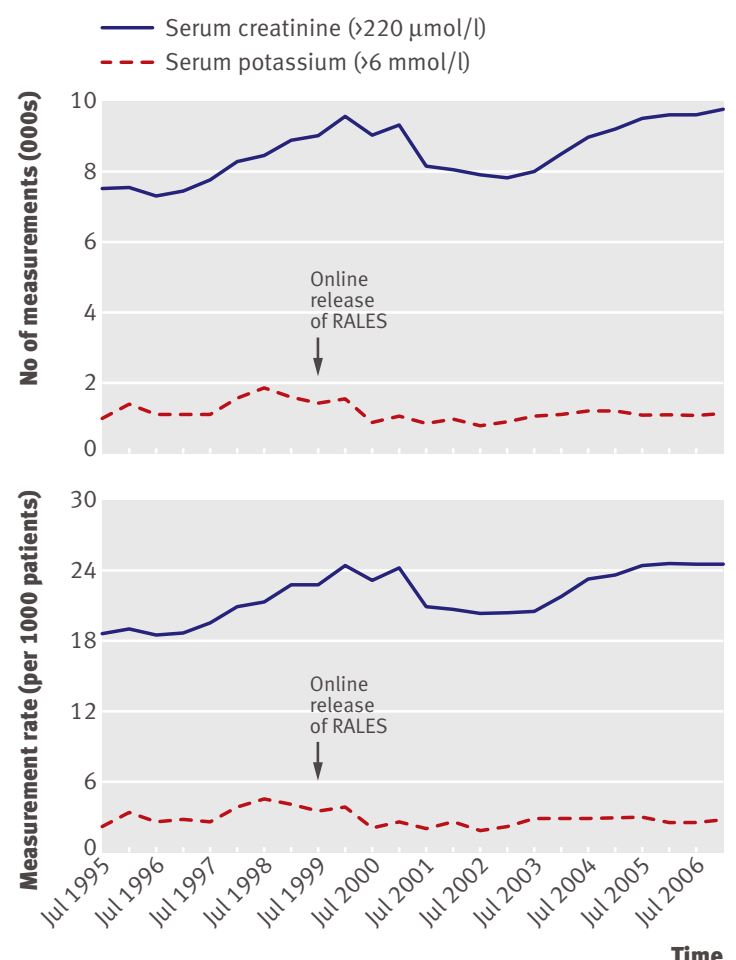

Fig 2 Serum measurements of creatinine/potassium (top) and rates of measurements (bottom) in Tayside population between 1995 and 2007

$(\mathrm{SE}=0.18, \mathrm{P}<0.01)$ between spironolactone use and serum creatinine or potassium measurements. The numbers/rates of high serum creatinine measurements $(>220 \mu \mathrm{mol} / \mathrm{l}$ ) and of hyperkalaemia (serum potassium measurements $>6 \mathrm{mmol} / \mathrm{l})$ did not increase significantly after the publication of RALES (fig 2). However, the rate of mild hyperkalaemia (defined as serum potassium $>5 \mathrm{mmol} / \mathrm{l}$ but $\leq 6 \mathrm{mmol} / \mathrm{l}$ ) increased over the study period (see web extra figure), suggesting that mild hyperkalaemia was appropriately identified and managed to avoid development of clinically significant hyperkalaemia. Few hospital admissions for hyperkalaemia occurred over time, ranging from three in the first quarter of 1995 to two in the last quarter of 2001 and three in 2007; we found no significant trend for increased hyperkalaemia (trend test, $\mathrm{P}=0.12$ ) over the entire study period. We found a significant trend for serum creatinine measurements $>220 \mu \mathrm{mol} / 1$ $(\mathrm{P}<0.01)$ over the study period, but this trend did not mirror increased spironolactone use.

\section{Spironolactone use among patients with heart failure receiving ACE inhibitors}

Figure 3 shows spironolactone use and hyperkalaemia (defined as serum potassium $>6 \mathrm{mmol} / \mathrm{l}$ ) in patients recently admitted to hospital for heart failure who were receiving angiotensin converting enzyme (ACE) inhibitors. Use of spironolactone increased after the online release of the RALES results, from 19.8 per 100 patients in the first half of 1999 to 70.1 per 100 patients by the second half of $2001(\mathrm{P}<0.01)$ and 61.3
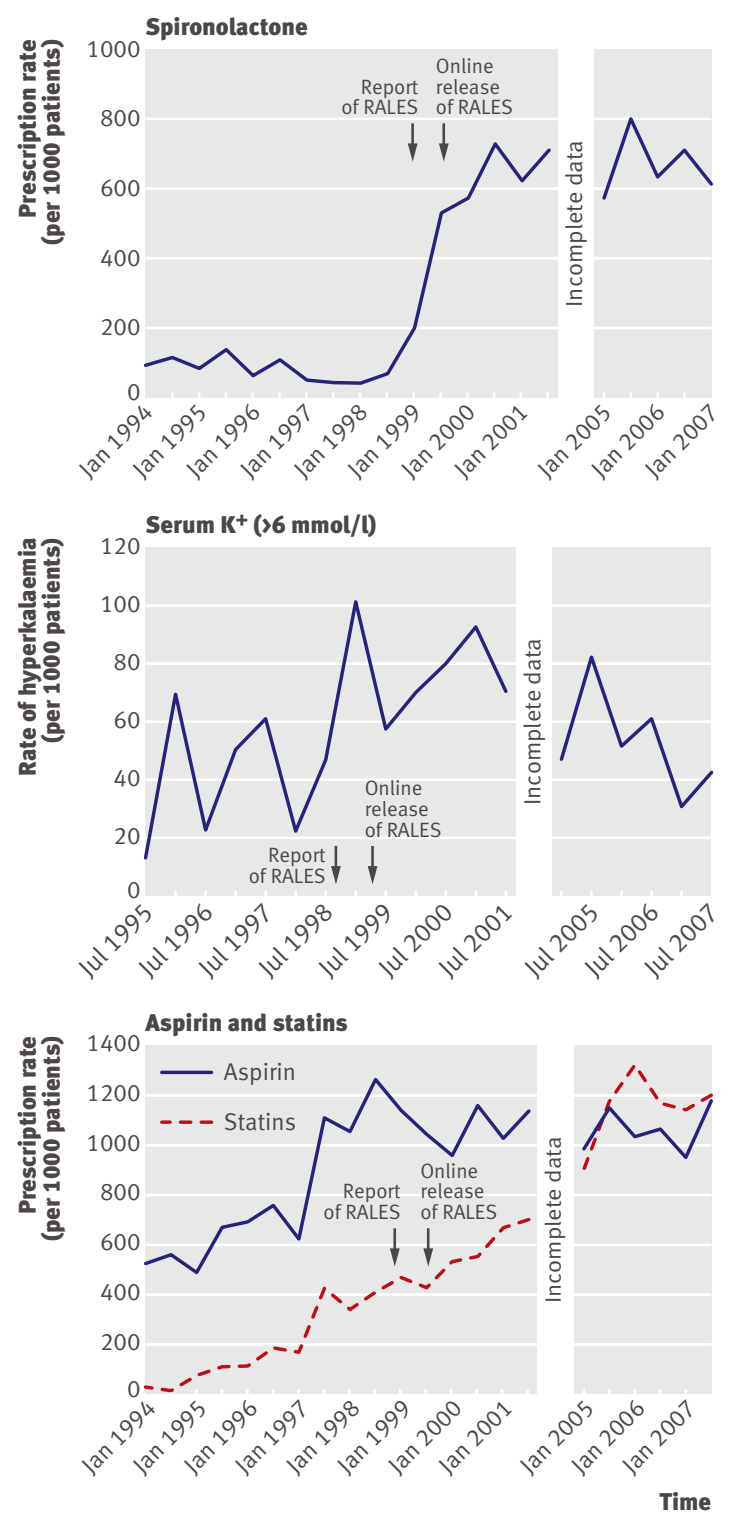

Fig 3 | Rates of spironolactone prescriptions and hyperkalaemia among patients recently admitted to hospital for heart failure who were receiving angiotensin converting enzyme inhibitors, and rates of aspirin and statins prescribing

in 2007. However, the rates of hyperkalaemia did not increase over time (from 9.9 per 100 patients in early 1999 to 6.9 per 100 patients by late $2001(\mathrm{P}<0.01)$ and 2.9 in 2007). We found no impact of the RALES study on use of aspirin and statins over time (fig 3). However, statin use rose gradually over time (trend test, $\mathrm{P}<0.01$ ), reflecting the impact of statins in the primary and secondary prevention of coronary heart disease. ${ }^{7-11}$

Characteristics of patients with spironolactone associated hyperkalaemia

Between 1995 and 2001, 578 patients had spironolactone associated hyperkalaemia (defined as serum potassium $>6 \mathrm{mmol} / \mathrm{l}$ ). The table shows the characteristics of these patients with heart failure, liver cirrhosis, and hypertension. We found no significant differences in sex or rates of hyperkalaemia between the three 
Characteristics of patients who developed spironolactone associated hyperkalaemia (serum $\mathrm{K}^{+}>6 \mathrm{mmol} / \mathrm{l}$ ) between 1995 and 2001. Values are numbers (percentages) unless stated otherwise

\begin{tabular}{|c|c|c|c|c|}
\hline Characteristics & $\begin{array}{l}\text { Heart failure } \\
\qquad(n=172)\end{array}$ & $\begin{array}{l}\text { Liver cirrhosis } \\
\quad(n=45)\end{array}$ & $\begin{array}{l}\text { Hypertension } \\
\qquad(n=361)\end{array}$ & $P$ value \\
\hline $\begin{array}{l}\text { Median (interquartile range) serum } \mathrm{K}^{+} \\
\text {concentration (mmol/l) }\end{array}$ & $6.4(6.2-6.7)$ & $6.4(6.2-6.7)$ & $6.4(6.2-6.8)$ & 0.889 \\
\hline \multicolumn{5}{|l|}{ Sex: } \\
\hline Men & $91(53)$ & $20(44)$ & $156(43)$ & \multirow{2}{*}{0.107} \\
\hline Women & $81(47)$ & $25(56)$ & $205(57)$ & \\
\hline Mean (SD) age & $73.2(9.7)$ & $55.6(12.5)$ & $72.8(12)$ & $<0.001$ \\
\hline \multicolumn{5}{|l|}{ Social deprivation: } \\
\hline 1 & $11(6)$ & $3(7)$ & $16(4)$ & \multirow{6}{*}{$<0.001$} \\
\hline 2 & $37(22)$ & $8(18)$ & $75(21)$ & \\
\hline 3 & $37(22)$ & $7(16)$ & $108(30)$ & \\
\hline 4 & $27(16)$ & $9(20)$ & $78(22)$ & \\
\hline 5 & $19(11)$ & $3(7)$ & $35(10)$ & \\
\hline 6 and 7 & $41(24)$ & $15(33)$ & $48(13)$ & \\
\hline \multicolumn{5}{|l|}{ Spironolactone daily dose: } \\
\hline$\leq 25 \mathrm{mg}$ & $61(36)$ & 0 & $90(25)$ & \multirow{3}{*}{$<0.001$} \\
\hline $26-50 \mathrm{mg}$ & $49(29)$ & $5(11)$ & $117(32)$ & \\
\hline$>50 \mathrm{mg}$ & $62(36)$ & $40(89)$ & $154(43)$ & \\
\hline Creatinine concentration >220 $\mu \mathrm{mol} / \mathrm{l}$ & $130(76)$ & $32(71)$ & $192(53)$ & $<0.001$ \\
\hline
\end{tabular}

groups of patients. Patients in the liver cirrhosis group were younger and more socially deprived and had used higher doses of spironolactone compared with patients in the heart failure and hypertension groups. The proportions with high creatinine $(>220 \mu \mathrm{mol} / \mathrm{l})$ at any time before the date of hyperkalaemia during the same period were $76 \%(\mathrm{n}=130)$ in the heart failure group, $71 \%(\mathrm{n}=32)$ in the liver cirrhosis group, and $53 \%(\mathrm{n}=192)$ in the hypertension group $(\mathrm{P}<0.01)$, indicating that renal impairment is a potential risk factor and presages hyperkalaemia associated with spironolactone use. Of these 578 patients, $451(78.0 \%)$ stopped taking spironolactone and $33(5.7 \%)$ reduced the dose during the follow-up period.

\section{DISCUSSION}

We saw an increased use of spironolactone in patients with heart failure after the report of the RALES study results in late 1998. This trend continued after the online release of RALES, which is consistent with the findings from a Canadian study that showed an increase in the spironolactone prescription rate from 34 per 1000 patients in 1994 to 149 per 1000 patients by late 2001.5 The use of spironolactone also markedly increased in patients with hypertension, but this increase pre-dated the RALES results reflecting a local research interest in spironolactone use in hypertension and the British Hypertension Society guidelines. ${ }^{212-15}$ Concurrent with this increased use of spironolactone, measurement of serum potassium and creatinine also increased, suggesting that patients who were at risk from spironolactone use were monitored (and presumably managed) well. In support of this concept, we found an increase in mild hyperkalaemia but no increase in more severe hyperkalaemia. Perhaps as a result of this, serious renal side effects were rare.
Our data support the view that proper monitoring in the community improves care and delivers maximum benefit to patients. ${ }^{16}$ Unlike the Canadian study ${ }^{5}$ which reported a considerable increase in the rate of hospital admission for hyperkalaemia, we did not see such an increase; nor did we see an increase in severe biochemical hyperkalaemia over time. Our results suggest that the benefit of spironolactone in patients with heart failure and hypertension can be realised in a "real world" population if this drug is carefully monitored. This highlights the usefulness of monitoring as a risk management tool and shows that proper monitoring can deliver substantial benefits for patients. This is an important observation, as a recent study showed that between 2005 and 2007 less than a third of eligible patients admitted to hospital for heart failure and participating in a quality improvement registry received aldosterone antagonist treatment as recommended by heart failure guidelines. ${ }^{17}$

The RALES study results had a major impact on use of spironolactone in patients with heart failure. ${ }^{1819} \mathrm{In}$ parallel, the 2004 British Hypertension Society guidelines recommended that spironolactone should be used as fourth line treatment and also resulted in a marked increase in the use of this drug, often added for patients already taking polytherapy. ${ }^{20}$ More recently, studies have shown the benefit of spironolactone in patients with and without hyperaldosteronism and patients with resistant hypertension. ${ }^{421}$ Increased aldosterone concentrations within the physiological range predisposed to the development of hypertension..$^{22}$ Renewed interest in mineralocorticoid antagonism thus exists.

Among patients who had spironolactone associated hyperkalaemia, $75 \%$ were over 65 years old, $61 \%$ had high serum creatinine before the development of hyperkalaemia, and $44 \%$ were taking a high daily dose of spironolactone. This highlights the need to monitor this group of patients even more carefully, as previous studies have shown that older patients are at higher risk of spironolactone associated hyperkalaemia. ${ }^{23-25}$

Our study has limitations. Firstly, the results reflect only one NHS region. However, we would expect other regions of the NHS to have similar monitoring systems. Secondly, we used ICD-9 and ICD-10 codes for hospital admission due to heart failure and liver disease, which may be subject to some degree of misclassification because of changes in coding patterns. Thirdly, this is an observational study. We did not adjust for any other risk factors, and we cannot rule out residual confounding. Further study on this topic in a large population would strengthen the evidence of safe spironolactone use in patients with heart failure.

\section{Conclusion}

The risk associated with use of spironolactone can be managed in the setting of the NHS, and adverse effects can be minimised. Our data provide reassurance that spironolactone is a safe drug in heart failure, hypertension, and liver disease when it is used in conjunction with appropriate monitoring. 


\section{WHAT IS ALREADY KNOWN ON THIS TOPIC}

Spironolactone is efficacious in patients with heart failure, resistant hypertension, and liver cirrhosis with secondary aldosteronism

A Canadian study found an alarming rate of hyperkalaemia due to spironolactone use in patients with heart failure

Monitoring patients at risk of adverse drug reactions can improve the benefit/risk ratio

\section{WHAT THIS STUDY ADDS}

No increased hyperkalaemia or renal toxicity occurred in association with increased use of spironolactone in patients with heart failure, hypertension, or liver disease

Spironolactone prescribing seems to be safe in the setting of the NHS, probably owing to careful monitoring of electrolytes and renal function
6 Wei L, Parkinson J, MacDonald TM. The Tayside Medicines Monitoring Unit (MEMO). In: Strom BL, ed. Pharmacoepidemiology. 4th ed. John Wiley and Sons, 2005:323-36.

7 Pedersen TR, Kjekshus J, Berg K, Haghfelt T, Faergeman O, Thorgeirsson G, et al. Randomised trial of cholesterol lowering in 4444 patients with coronary heart disease: the Scandinavian Simvastatin Survival Study (4S). Lancet 1994;344:1383-9.

8 Sacks FM, Pfeffer MA, Moye LA, Rouleau JL, Rutherford JD, Cole TG, et al. The effect of pravastatin on coronary events after myocardial infarction in patients with average cholesterol levels. N Engl J Med 1996;335:1001-9.

9 The Long-Term Intervention with Pravastatin in Ischaemic Disease (LIPID) Study Group. Prevention of cardiovascular events and death with pravastatin in patients with coronary heart disease and a broad range of initial cholesterol levels. N Engl J Med 1998;339:1349-57.

10 GISSI Prevenzione Investigators (Gruppo Italiano per lo Studio della Sopravvivenza nell'Infarto Miocardico). Results of the low-dose $(20 \mathrm{mg})$ pravastatin GISSI Prevenzione trial in 4271 patients with recent myocardial infarction: do stopped trials contribute to overall knowledge? Ital Heart / 2000;1:810-20.

11 Cholesterol Treatment Trialists' Collaborators. Efficacy and safety of cholesterol-lowering treatment: prospective meta-analysis of data from 90056 participants in 14 randomised trials of statins. Lancet 2005;366:1267-78.

Contributors: $\mathrm{LW}$ did the statistical analysis and wrote the first draft of the paper. All authors were involved in the design of the study, interpretation of results, and re-drafting of the paper. LW is the guarantor. Funding: This study was funded by the TENOVUS Scotland (T06/44). LW holds a special training fellowship in health services and health of the public research award from the UK Medical Research Council (G106/ 1249). The funding body did not have any role in study design, data analysis, result interpretations, or report submission.

Competing interests: All authors have completed the Unified Competing Interest form at www.icmje.org/coi_disclosure.pdf (available on request from the corresponding author) and declare (1) no financial support from any commercial company for the submitted work; (2) TMM received consultancy fees, honoraria, and travel expenses in the past three years from Pfizer, Servier, Novartis, Wyeth, Kaiser Permanante, Takeda, Recordati, NiCox, Quintiles, and Speedel; ADS received consultancy fees, honoraria, and travel expenses from Pfizer in the past three years; ADW received consultancy fees, honoraria, and travel expenses from Servier and Pfizer in the past three years; (3) no spouses, partners, or children with relationships with commercial entities that might have an interest in the submitted work; and (4) no non-financial interests that may be relevant to the submitted work.

Ethical approval: This study was approved by Tayside Research Ethics Committee and the Tayside Caldicott Guardians.

Data sharing: No additional data available.

1 Pitt B, Zannad F, Remme WJ, Cody R, Castaigne A, Perez A, et al. The effect of spironolactone on morbidity and mortality in patients with severe heart failure. N Engl J Med 1999;34:709-17.

2 Lim PO, Jung RT, MacDonald TM. Raised aldosterone to renin ratio predicts antihypertensive efficacy of spironolactone: a prospective cohort follow-up study. Br J Clin Pharmacol 1999;48:756-60.

3 Ouzan J, Pérault C, Lincoff AC, Carré E, Mertes M. The role of spironolactone in the treatment of patients with refractory hypertension. Am J Hypertens 2002;15:333-9.

4 Chapman N, Dobson J, Wilson S, Dahlöf B, Sever PS, Wedel H, et al. Effect of spironolactone on blood pressure in subjects with resistan hypertension. Hypertension 2007;49:839-45.

5 Juurlink DN, Mamdani MM, Lee DS, Kopp A, Austin PC, Laupacis A, et al. Rates of hyperkalaemia after publication of the Randomized Aldactone Evaluation Study. N Engl J Med 2004;351:543-51.

12 Lim PO, Rodgers P, Cardale K, Watson A, MacDonald TM. Potentially high prevalence of primary aldosteronism in a general practice population in Tayside, Scotland. Lancet 1999;353:40.

13 Lim PO, Jung RT, MacDonald TM. Is aldosterone the missing link in refractory hypertension? Aldosterone to renin ratio as a marker of inappropriate aldosterone activity. J Hum Hypertens 2002;16:153-8.

14 Lim PO, Young W Jr, MacDonald TM. A review of the medical treatment of primary aldosteronism. J Hypertens 2001;19:353-61.

15 Lim PO, Struthers AD, MacDonald TM. The neuro-hormonal natural history of essential hypertension: towards primary or tertiary hyperaldosteronism? J Hypertens 2002;20:11-5.

16 Glasziou P, Irwig L, Mant D. Monitoring in chronic disease: a rational approach. BMJ 2005;330:644-8.

17 Albert NM, Yancy CW, Liang L, Zhao X, Hernandez AF, Peterson ED, et al. Use of aldosterone antagonists in heart failure. JAMA 2009;302:1658-65

18 Verhamme K, Mosis G, Dieleman J, Stricker B, Sturkenboom M. Spironolactone and risk of upper gastrointestinal events: population based case-control study. BMJ 2006;333:330.

19 Masoudi FA, Gross CP, Wang Y, Rathore SS, Havranek EP, Foody JM, et al. Adoption of spironolactone therapy for older patients with heart failure and left ventricular systolic dysfunction in the United States, 1998-2001. Circulation 2005;112:39-47.

20 Williams B, Poulter NR, Brown MJ, Davis M, McInnes GT, Potter JF, et al. British Hypertension Society guidelines for hypertension management 2004 (BHS-IV): summary. BMJ 2004;328:634-40

21 Calhoun DA. Use of aldosterone antagonists in resistant hypertension. Prog Cardiovasc Dis 2006;48:387-96.

22 Vasan RS, Evans JC, Larson MG, Wilson PW, Meigs JB, Rifai N, et al. Serum aldosterone and the incidence of hypertension in nonhypertensive persons. N Engl J Med 2004;351:33-41.

23 Obialo $\mathrm{Cl}$, Ofili EO, Mirza T. Hyperkalemia in congestive heart failure patients aged 63 to 85 years with subclinical renal disease. $A m$ J Cardiol 2002;90:663-5.

24 Schepkens H, Vanholder R, Billiouw JM, Lameire N. Life-threatening hyperkalemia during combined therapy with angiotensin-converting enzyme inhibitors and spironolactone: an analysis of 25 cases. Am J Med 2001;110:438-41.

25 Svensson M, Gustafsson F, Galatius S, Hildebrandt PR, Atar D. Hyperkalaemia and impaired renal function in patients taking spironolactone for congestive heart failure: retrospective study. $B M J$ 2003;327:1141-2.

Accepted: 9 February 2010 